\title{
TOXICIDADE DOS INSETICIDAS QUÍMICOS SOBRE ESPÉCIES DE ISARIA
}

Rosineide da Silva Lopes ${ }^{1 *}$, Geiziquele de Lima², Luciana Gonçalves de Oliveira ${ }^{1}$, Antonio Félix da Costa ${ }^{1}$, Maria Tereza dos Santos Correia ${ }^{3}$,

Elza Áurea de Luna Alves Lima4, Vera Lúcia de Menezes Lima³.

\begin{abstract}
${ }^{1 *}$ Instituto Agronômico de Pernambuco/IPA, Av. General San Martin, Bongi - CEP 50.7610-000, Recife, PE, Brasil, Fone: + 5581 3184-7366, rs.lopes@hotmail.com. Dra. em Ciências Biológicas/UFPE. Autor para correspondência. ${ }^{2}$ Instituto de Ciências Naturais Humanas e Sociais, Universidade Federal de Mato Grosso/UFMT, Snop, MT, Brasil. ${ }^{3}$ Departamento de Bioquímica, Universidade Federal de Pernambuco/UFPE, Recife, PE, Brasil. ${ }^{4}$ Departamento de Micologia, Universidade Federal de Pernambuco/UFPE, Recife, PE, Brasil.
\end{abstract}

\section{RESUMO}

O controle de insetos-praga é usualmente realizado com produtos químicos, os quais podem contaminar o homem e o meio ambiente. $O$ trabalho teve por objetivo avaliar o efeito tóxico dos inseticidas clorpirifós, acetamiprid, thiamethoxan e lambacyhalothrin sobre espécies de Isaria, visando à utilização destes agentes biológicos no controle integrado de insetos-praga. Os inseticidas foram adicionados ao meio Ágar Sabouraud e à solução Tween $80(0,1 \%)$, em três concentrações preestabelecidas de acordo com as recomendações do fabricante. Em seguida, analisado o efeito dos inseticidas sobre a germinação, crescimento micelial e esporulação das linhagens, sendo utilizado o Índice Biológico (IB) para classificar a toxicidade dos inseticidas. Estes foram classificados como compatíveis às linhagens, exceto clorpirifós, que apresentou IB moderadamente tóxico a tóxico. Os dados demonstraram que os inseticidas podem ser associados às espécies de Isaria, e testados no controle de insetos-praga em áreas agrícolas e urbanas.

PALAVRA-CHAVE: inseticidas, fungos entomopatogênicos, controle de insetos. 


\section{ABSTRACT}

\section{TOXICITY OF CHEMICAL INSECTICIDES USED IN TERMITE CONTROL ON ISARIA SPECIES}

Pest insect control is carried out with chemicals, which can contaminate man and the environment. The objective of this work was to evaluate the effect of the insecticides chlorpyrifos, acetamiprid, thiamethoxan and lambacyhalothrin on Isaria species, aiming at the use of the biological agents in the integrated control of pest insect. The insecticides were added to the Sabouraud Agar medium and the Tween 80 solution $(0.1 \%)$ at three pre-established concentrations according to the manufacturer's recommendations. Afterwards, the insecticide on the germination, mycelial growth and sporulation of the strains was analyzed, and the Biological Index (IB) was used to classify the toxicity of the insecticides. These were classified as compatible with strains except chlorpyrifos, which presented moderate to toxic IB. The data demonstrate that insecticides can be associated with /saria species and tested for pest insect control in agricultural and urban areas.

KEYWORDS: insecticide, termite, entomopathogenic fungi, insect control.

A partir da conscientização mundial sobre os riscos na utilização dos inseticidas químicos e a necessidade de se reduzir o uso destes, a procura de produtos eficientes no controle de pragas que não causam impacto no ambiente, em especial os microrganismos, tem sido cada vez maior (DALZOTO; UHRY, 2009). O uso de inseticidas químicos no controle de insetos-praga pode causar o desequilíbrio ambiental, doenças em seres humanos e em outros animais, desenvolvimento de mecanismos de resistência em insetos (ALVES, 1998; POURSEYED et al., 2010).

O controle biológico, por meio de fungos entomopatogênicos tem se mostrado uma alternativa eficiente $\mathrm{e}$ segura no controle de insetos-praga, pois mantém as populações de insetos em equilíbrio, diminuindo o processo de reprodução sem ocasionar danos a outros animais e ao meio ambiente (CHOUVENC et al., 2011; Lv et al., 2011; SABBOUR; ABDEL-RAHMAN, 2013).

Isaria está entre os gêneros de fungos entomopatogênicos mais utilizados em programas de controle biológico, sendo empregado na Europa e nas Américas do Norte e Latina no controle de insetos (ALVES et al., 2008). Relatos na literatura mostram a eficiência das espécies Isaria farinosa (Holm: Fries) Fries, Isaria fumosorosea (Wize) Brown \& Smith e Isaria javanica (Frieder \& Bally) Samson \&. Hywell-Jones no controle de insetos-praga como: Diaphorina citri Kuwayama, Coptotermes gestroi (Wasmann), 
Planococus citri (Risso) Coptotermes formosanus Shiraki, Nasutitermes corniger (Motschulsky) entre outros (MEYER et al., 2008; LOPES et al., 2011; DEMIRCI et al., 2011, WRIGHT; ALAN, 2013; PASSOS et al., 2014; LOPES et al., 2017).

A associação dos fungos entomopatogênicos com outras técnicas de controle, como o uso de inseticidas é viável e provavelmente pode aumentar a eficiência destes, no controle de insetos-praga, além de, reduzir os prejuízos causados ao meio ambiente (DEL-BELLO et al., 2001; AMBETHGAR, 2009). Dessa forma, esse trabalho teve por objetivo avaliar a compatibilidade dos inseticidas (clorpirifós, acetamiprid, thiamethoxan e lambacyhalothrin) com as linhagens de I. farinosa, I. fumosorosea e I. javanica, visando à utilização destes no controle integrado de insetos-praga.

Os experimentos foram conduzidos no Laboratório de Controle Biológico do Instituto Agronômico de Pernambuco/IPA. Foram testadas cinco linhagens de três espécies de Isaria. I. farinosa (URM5016 e URM5060) e I. javanica URM4995 foram obtidas pela Coleção de Cultura URM (WDCM604) da Universidade Federal de Pernambuco (UFPE), enquanto I. farinosa ESALQ1205 e $l$. fumosorosea ESALQ1296 foram cedidas pela Coleção de Microrganismos da Escola Superior de Agricultura Luiz de Queiroz - ESALQ da Universidade de São Paulo (USP). A viabilidade das linhagens foi determinada pelos percentuais de germinação. Em seguida, as linha- gens foram cultivadas em Meio Sabouraud (Peptona-Dextrose-Agar) durante 12 dias; após esse período, $10 \mathrm{~mL}$ de Tween 80 a 0,1\%, foram adicionados aos conídios de cada linhagem, a suspensão foi quantificada em câmera de Neubauer, e a concentração foi ajustada para $1 \times 10^{7}$ conídios $/ \mathrm{mL}$. Os inseticidas químicos selecionados para os experimentos foram clorpirifós, acetamiprid, thiamethoxan e lambacyhalothrin. Estes foram utilizados em três concentrações preestabelecidas de acordo com as recomendações do fabricante, sendo a concentração média (CMed) aquela recomendada para o emprego no campo, a concentração mínima (CMed/2) e a concentração máxima (CMedx2), conforme a Tabela 1. $\mathrm{O}$ efeito dos inseticidas foi avaliado por meio da germinação, crescimento vegetativo e esporulação dos fungos, cujos ensaios foram realizados em cinco repetições. Para avaliação da germinação, os produtos químicos foram adicionados aos tubos de ensaio contendo a solução Tween $(0,1 \%)$ e a solução Tween sem os inseticidas foi utilizada como controle. Em seguida, $1 \mathrm{~mL}$ da suspensão $\left(1 \times 10^{8}\right.$ conídios $/ \mathrm{mL}$ ) das linhagens foi inoculado em $9 \mathrm{~mL}$ de solução Tween 80 para a obtenção de suspensões com $1 \times 10^{7} \mathrm{co}-$ nídios $/ \mathrm{mL}$, após uma hora foi inoculado $0,1 \mathrm{~mL}$ das suspensões, separadamente, em placas de Petri contendo $S A B$ e incubadas em BOD $\left(26 \pm 1^{\circ} \mathrm{C}\right.$ e $80 \pm 10$ $\%$ UR). A germinação foi determinada após 16 horas, por meio da observação de 500 conídios (entre germinados e não germinados), sendo o percentual de ger- 
minação calculado pela fórmula $(G=n x$ 100/500), conforme descrito por ALVES; PEREIRA (1998). Para o crescimento e a esporulação, os inseticidas foram adicionados ao SAB líquido $\left(45^{\circ} \mathrm{C}\right)$, nas concentrações mínima, média e máxima, e o meio foi vertido em placas de Petri esterilizadas. Em seguida, discos de 0,3 $\mathrm{mm}$ de papel filtro com $0,01 \mathrm{~mL}$ da suspensão $\left(1 \times 10^{7}\right.$ conídios $\left./ \mathrm{mL}\right)$ das cinco linhagens de Isaria foram inoculados separadamente, em placas de Petri com $\mathrm{SAB}$, acrescidas das concentrações dos inseticidas e sem os produtos químicos (controle). As placas foram incubadas em BOD $\left(26 \pm 1^{\circ} \mathrm{C}\right.$ e $80 \pm 10 \%$ UR $)$ por 12 dias, sendo o crescimento micelial determinado pelo diâmetro da colônia. Para avaliar a esporulação fúngica, fragmentos dessas colônias foram transferidos para um tubo de ensaio contendo $10 \mathrm{~mL}$ de solução Tween 80 (0,1\%). A suspensão foi agitada por aproximadamente dois minutos em Vortex e os esporos foram quantificados em câmara de Neubauer. O efeito fungitóxico dos inseticidas foi medido pelo Índice Biológico, obtido com por meio da fórmula $\mathrm{IB}=47[\mathrm{CV}]+43[\mathrm{ESP}]+10[\mathrm{GERM}] / 100$, onde CV: porcentagem de crescimento vegetativo com relação à testemunha; ESP: porcentagem de esporulação com relação à testemunha; GERM: porcentagem de germinação dos conídios em relação à testemunha. O valor do IB pode variar de: 0-41 (tóxico), 42-66 (moderadamente tóxico) e > 66 (compatível) (ROSSI-ZALAF et al., 2008).
Conforme o valor do Índice Biológico (IB), os inseticidas foram considerados compatíveis com os fungos, exceto clorpirifós, classificado como moderamente tóxico e tóxico para as linhagens testadas, principalmente nas concentrações média e máxima do inseticida (Tabela 1). Resultados similares foram envidenciados por BATISTA-FILHO et al. (2001) que constataram a compatibilidade do inseticida thiamethoxan quando analisado nas concentrações mínimas e máxima sobre I. farinosa. Da mesma forma, os produtos químicos testados foram compatíveis com Isaria sp., nas concentrações (CR= recomenda pelo fabricante, $1 / 2 \mathrm{CR}, 2 \mathrm{CR}$ ), sendo que todos os produtos apresentaram IB maior que 66 (PURETZ et al., 2012). Do mesmo modo, BERNAL et al. (2014) avaliaram o efeito dos fungicidas e inseticidas sobre I. fumosorosea, em três concentrações (FR = dose recomendada para campo, 0,5 x FR e 0,25 x FR) e observaram que os produtos benlate, carboxin captan,metalaxil-mancozeb e mancozeb foram tóxicos e o thiamethoxan foi classificado como compatível ao fungo.

Estudos prévios mostraram a toxicidade de produtos biológicos e químicos sobre outros fungos. PIRES et al. (2010) avaliaram o efeito de seis inseticidas usados no controle de Tuta absoluta (Meyrick), sobre linhagens de Metarhizium anisopliae (Metsch.) Sorokin e observaram que o inseticida biológico Espinosa (três concentrações) apresentou IB compatível, enquanto o $\mathrm{Nim}$ foi compatível e tóxico com o aumento das 
concentrações. Resultados semelhantes foram encontrados por SOARES; MONTEIRO (2011) quando analisaram a ação dos carrapaticidas químicos usados contra Riphicephalus (Boophilus) microplus (Canestrini) sobre M. anisopliae, e verificaram que os produtos cipermetina e espinosade apresentaram IB compatível com o entomopatógeno. Da mesma forma, seis produtos utilizados no controle de Diaphorina citri Kuwayama foram classificados como moderamente tóxico e tóxico sobre Beauveria bassiana (Bals.) Vuillemin, especialmente os produtos thiamethoxan, nim e malationa (PINTO et al., 2012). SANTOS et al.
(2015) verificaram que extrato aquoso de Ricinus communis L. e Poincianella pyramidalis Queiroz, nas concentrações 5, 10 e $20 \%$, apresentaram IB compatível para os isolados Fusarium incarnatum-equiseti species complex (FIESC), enquanto o extrato hidroetílico foi classificado com moderamente tóxico e tóxico para a maioria dos isolados.

Os inseticidas foram compatíveis com as linhagens fúngicas, exceto o produto clorpirifós, sendo possível utilizá-los em associação com os fungos no controle de insetos-praga em áreas agrícolas e urbanas. 


\section{REFERÊNCIAS}

ALVES, S.B. Fungos entomopatogenicos. In: . Controle microbiano dos insetos. 2 th. Ed. Piracicaba: FEALQ, 1998. chap.11, p. 289-370.

ALVES, S.B.; PEREIRA, R.M. Produção de fungos entomopatogênicos. In: ALVES, S.B. (Ed). Controle microbiano de insetos. 2. Ed. Piracicaba: FEALQ, 1998. cap. 27. p. 845-869.

ALVES, S.B.; LOPES, R.B.; VIEIRA, S.A.; TAMAI, M.A. Fungos Entomopatogênicos usados no controle de pragas na América Latina. In: ALVES, S.B.; LOPES, R.B. (Ed.). Controle Microbiano de Pragas na América Latina: avanços e desafios. Piracicaba: FEALQ, 2008. cap. 3, p. 69-110.

AMBETHGAR, V. Potential of entomopathogenic fungi in insecticide resistance management (IRM): A review. Journal of Biopesticides, v. 2, n. 2, p. 177-193, 2009.

BATISTA-FILHO, A.; ALMEIDA, J.E.M.; LAMAS, C. Effect of thiamethoxam on entomopathogenic microorganisms. Neotropical Entomology, v. 30, n. 3, p. 437-447, 2001.

BERNAL, E.P.G.; ÁlVAREZ, M.I.G.; MOGOLLÓN, M.V.Z. In vitro Compatibilidad Isaria fumosorosea (Wize) Brown y Smith (Hypocreales: Clavicipitaceae) con plaguicidas comerciales. Acta Agronómica, v. 63, n. 1, p. 48-54, 2014.

CHOUVENC, T.; SU, N.Y.; KENNETH, G.J. Fifty of attempted biological control of termites - analysis a failure. Biological Control, v. 59, n. 2., p. 69-82, 2011.

DAL-BELLO, G.; PADIN, S.; LÓPES-LASTRA, C.; FABRIZIO, M. Laboratory evaluation of chemical-biological control of the rice weevil (Sitophilus oryzae L.) in stored grains. Journal of Stored Products Research, v. 37, n. 1, p.77-84, 2001.

DALZOTO, P.R.; UHRY, K.F. Controle biológico de pragas no Brasil por meio de Beauveria bassiana (Bals.) Vuill. Biológico, v. 71, p. 37-41, 2009.

DEMIRCI, F., MUST, M., KAYDAN, M. B., ULGENTUR, S. 2011. Effects of some fungicides on Isaria farinosa, and in vitro growth and infection rate on Planococcus citri. Phytoparasitica, v. 39, n. 4, p. 353-360, 2011.

LOPES, R. S.; SVEDESE, V. M.; PORTELA, A.P.A.S.; ALBUQUERQUE, A.C.; LUNAALVES LIMA, E.A.Virulence and biological aspects of Isaria javanica (Frieder \& Bally) Samson \& Hywell-Jones in Coptotermes gestroi (Wasmann) (Isoptera: Rhinotermitidae). Arquivos do Instituto Biológico, v. 78, n. 4, p. 565-572, 2011.

LOPES, R. S., LIMA, G., M.T.S., COSTA, A.F, LUNA-ALVES LIMA, E.A., LIMA, V.L.M. The potential of Isaria spp. as a bioinsecticide for the biological control of Nasutitermes corniger. Biocontrol Science and Technology, v. 27, n. 9, p. 1038-1048, 2017.

LV, J.; WILSON, L.T.; BEUZELIN, J. M.; WHITE, W.H.; REAGAN, T.E.; WAY, M.O. Impact of Cotesia flavipes (Hymenoptera: Braconidae) as an augmentative biocontrol agent for the sugarcane borer (Lepidoptera: Crambidae) on rice. Biological Control, 56, n. 2, p. 159-169, 
2011.

MEYER, J.M.; HOY, M.A.; BOUCIAS, D.G. Isolation and characterization of an Isaria fumosorosea isolate infecting the Asian citrus psyllid in Florida. Journal of Invertebrate Pathology, v. 99, n. 1, p. 96-102, 2008.

PASSOS, E.M.; ALBUQUERQUE, A.C.; MARQUES, E.J.; TEIXEIRA, V.W.; SILVA, C.C.M.; OLIVEIRA, M. A.P. Efeitos de isolados do fungo Isaria (Persoon) sobre o cupim subterrâneo Coptotermes gestroi (Wasmann) (Isoptera: Rhinotermitidae). Arquivos do Instituto Biológico, v. 81, n. 3, p. 232-237, 2014.

PINTO, A.P.F.; BATISTA-FILHO, A; ALMEIDA, J.E.M.; WENZEL, I.M. Patogenicidade de Beauveria bassiana ao psilídeo Diaphorina citri e compatibilidade do fungo com produtos fitossanitários. Pesquisa Agropecuária Brasileira, 47, n. 12, p. 1673-1680, 2012.

PIRES, L.M. MARQUES, E. J.; OLIVEIRA, J.V.; ALVES, S.B. 2010. Seleção de isolados de fungos entomopatogênicos para o controle de Tuta absoluta (Meyrick) (Lepidoptera: Gelechiidae) e sua compatibilidade com alguns inseticidas usados na cultura do tomateiro. Neotropical Entomology, v. 39, n. 6, p. 977-984, 2010.

POURSEYED, S. H.; TAVASSOLI, M.; BERMOUSI, I.; MARDANI, K. Metarhizium anisopliae (Ascomycota: Hypocreales): An effective alternative to chemical acaricides against different developmental stages of fowl tick Argas persicus (Acari: Argasidae). Veterinary Parasitology, v. 172, n. 4, p. 305-310, 2010.

ROSSI-ZALAF, L.S. ALVES, S.B.; LOPES, R.B.; NETO S.S. TANZINI, M.R. (2008). Interação de microrganismos com outros agentes de controle de pragas e doenças. In: ALVES, S.B., LOPES, R.B. (Eds). Controle Microbiano de Pragas na América Latina: Avanços e Desafios. Ed Piracicaba: FEALQ, 2008. Cap. p.279- 302.

SABBOUR M.M.; ABDEL-RAHMAN, A. Efficacy of isolated Nomuraea rileyi and Spinosad against corn pests under laboratory and field conditions in Egypt. Annual Research \& Review in Biology, v. 3, n. 4, p. 903-912, 2013.

SANTOS, A.C.S.; OLIVEIRA, R.L.S.; COSTA, A.F.; TIAGO, P.V.; OLIVEIRA, N.T. Controlling Dactylopius opuntiae with Fusarium incarnatum - equiseti species complex and extracts of Ricinus communis and Poincianella pyramidalis. Journal of Pest Science, v. 88, n. 2, p. 539547, 2015. doi:10.1007/s10340-015-0689-4

SOARES, F.B.; MONTEIRO, A.C. Compatibilidade de Metarhizium anisopliae com carrapaticidas químicos. Arquivos do Instituto Biológico, v. 78, n. 3, p. 385-391, 2011.

WRIGHT, M., ALAN, L. Combined effect of microbial and chemical control agents on subterranean termites. Journal of Microbiology, v. 51, n. 5, p. 578-58, 2013. 
Tabela1. Compatibilidade dos inseticidas químicos sobre as espécies de Isaria.

Os valores do IB podem variar: 0-41 (tóxico - T), 42-66 (moderadamente tóxico -MT) e 66 (compatível - C), segundo ROSSI-ZALAF et al., 2008.

\begin{tabular}{lccccc} 
Inseticida/Concentração & \multicolumn{5}{c}{ Linhagens/lB Classificação } \\
\cline { 2 - 6 } & $\begin{array}{l}\text { I. farnosa } \\
\text { ESALQ1205 }\end{array}$ & $\begin{array}{l}\text { I. farinosa } \\
\text { URM5016 }\end{array}$ & $\begin{array}{l}\text { I. farinosa } \\
\text { URM5060 }\end{array}$ & $\begin{array}{l}\text { I. javanica } \\
\text { URM4995 }\end{array}$ & $\begin{array}{c}\text { I. fumosorosea } \\
\text { ESALQ1926 }\end{array}$ \\
Corpirifós & & & & & \\
Mínima: 0,75 mL/L & $52,57 \mathrm{MT}$ & $81,05 \mathrm{C}$ & $83,23 \mathrm{C}$ & $85,69 \mathrm{C}$ & $81,34 \mathrm{C}$ \\
Média: $1,50 \mathrm{~mL} / \mathrm{L}$ & $57,73 \mathrm{MT}$ & $60,71 \mathrm{MT}$ & $73,62 \mathrm{C}$ & $73,28 \mathrm{C}$ & $81,34 \mathrm{C}$ \\
Máxima: $3,0 \mathrm{~mL} / \mathrm{L}$ & $28,17 \mathrm{~T}$ & $55,92 \mathrm{MT}$ & $46,17 \mathrm{MT}$ & $32,00 \mathrm{~T}$ & $65,97 \mathrm{MT}$ \\
Acetamiprid & & & & & \\
Mínima: $0,125 \mathrm{~g} / \mathrm{L}$ & $95,26 \mathrm{C}$ & $88,68 \mathrm{C}$ & $96,93 \mathrm{C}$ & $93,71 \mathrm{C}$ & $94,13 \mathrm{C}$ \\
Média: $0,25 \mathrm{~g} / \mathrm{L}$ & $90,15 \mathrm{C}$ & $85,09 \mathrm{C}$ & $94,93 \mathrm{C}$ & $95,39 \mathrm{C}$ & $92,64 \mathrm{C}$ \\
Máxima: $0,5 \mathrm{~g} / \mathrm{L}$ & $81,05 \mathrm{C}$ & $81,57 \mathrm{C}$ & $90,33 \mathrm{C}$ & $87,26 \mathrm{C}$ & $885,9 \mathrm{C}$ \\
Thiamethoxan & & & & & \\
Mínima: $0,10 \mathrm{~g} / \mathrm{L}$ & $92,79 \mathrm{C}$ & $96,25 \mathrm{C}$ & $98,61 \mathrm{C}$ & $95,24 \mathrm{C}$ & $102,66 \mathrm{C}$ \\
Média: $0,20 \mathrm{~g} / \mathrm{L}$ & $96,59 \mathrm{C}$ & $91,38 \mathrm{C}$ & $94,78 \mathrm{C}$ & $95,25 \mathrm{C}$ & $97,10 \mathrm{C}$ \\
Máxima: $0,40 \mathrm{~g} / \mathrm{L}$ & $85,59 \mathrm{C}$ & $84,22 \mathrm{C}$ & $87,72 \mathrm{C}$ & $93,95 \mathrm{C}$ & $97,02 \mathrm{C}$ \\
Lambacyhalothrin & & & & & \\
Mínima: $0,50 \mathrm{~mL} / \mathrm{L}$ & $78,20 \mathrm{C}$ & $96,30 \mathrm{C}$ & $96,69 \mathrm{C}$ & $89,65 \mathrm{C}$ & $82,05 \mathrm{C}$ \\
Média: $1,0 \mathrm{~mL} / \mathrm{L}$ & $68,38 \mathrm{C}$ & $92,58 \mathrm{C}$ & $87,79 \mathrm{C}$ & $87,15 \mathrm{C}$ & $83,98 \mathrm{C}$ \\
Máxima: $2,0 \mathrm{~mL} / \mathrm{L}$ & $66,99 \mathrm{C}$ & $81,62 \mathrm{C}$ & $82,80 \mathrm{C}$ & $84,31 \mathrm{C}$ & $81,05 \mathrm{C}$ \\
\hline
\end{tabular}

Recebido em: 14/12/2017

Aprovado em: 03/06/2019 